Article

\title{
Relativistic Statistical Mechanics vs. Relativistic Thermodynamics
}

\section{Gonzalo Ares de Parga ${ }^{1, \star}$ and Benjamín López-Carrera ${ }^{2}$}

${ }^{1}$ Department of Physics, School of Physics and Mathematics, National Polytechnic Institute, U.P. Adolfo López Mateos, Zacatenco, C.P. 07738, México D.F., México

${ }^{2}$ School of Computing, National Polytechnic Institute, U.P. Adolfo López Mateos, Zacatenco, C.P. 07738, México D.F., México; E-Mail: beneleci@gmail.com

* Author to whom correspondence should be addressed; E-Mail: gadpau @ hotmail.com; Tel.: +52-55-5729-6000 ext. 55017; Fax: +52-55-5729-6000 ext. 55015.

Received: 30 June 2011; in revised form: 16 August 2011 / Accepted: 5 September 2011 / Published: 9 September 2011

\begin{abstract}
Based on a covariant theory of equilibrium Thermodynamics, a Statistical Relativistic Mechanics is developed for the non-interacting case. Relativistic Thermodynamics and Jüttner Relativistic Distribution Function in a moving frame are obtained by using this covariant theory. A proposal for a Relativistic Statistical Mechanics is exposed for the interacting case.
\end{abstract}

Keywords: relativistic statistical mechanics; covariant theory; jüttner distribution function; canonical transformation

\section{Introduction}

Some years after the Planck-Einstein [PE] [1,2] proposal about the relativistic transformations of equilibrium Thermodynamics, Jüttner [3] obtained his famous relativistic distribution function. Due to the Einstein doubts [4] about his own theory, Ott [5] presented a new theory which was fundamentally different to the PE one [1,2]. This last proposal represented the source of many other works which Balescu [6] has explained to differ from PE theory by a kind of gauge theory adapted to Relativistic Thermodynamics. Nevertheless, due to the lack of a covariant theory, the problem was still considered as an open problem during many years. Moreover, in 1998 Sieniutycz [7] still remarked that some 
authors concluded that equilibrium statistical mechanics cannot provide an unambiguous answer to the relativistic transformation formulae of thermodynamical quantities and, therefore, all of the three kinds of transformations, the PE [1,2], the Ott [O] [5] and the Landsberg [L] [8-10] proposals, are acceptable. Even more, nowadays Landsberg and Matsas [4,11] claimed the impossibility of the existence of relativistic transformation of the temperature. However, recently Ares de Parga et al. [12] have showed, by noticing that the internal energy must be redefined, that the existence of a relativistic transformation of the temperature is possible. Moreover, since the regular concept of internal energy has to be abandoned and substituted by a redefined energy, it is possible to obtain the invariance of the form of Thermodynamics. Starting by using the Redefined Relativistic Thermodynamics [RRT] an incipient non-covariant statistical theory has been developed by López-Carrera and Ares de Parga [13]. On the other hand, Nakamura [14] has proposed a variation to the van-Kampen theory [15] modified by Israel $[16,17]$ which correct the tensor character of it. In fact, due to the Nakamura's ideas, Ares de Parga and López-Carrera [18] have modified their theory, in a recent paper, in order to obtain a covariant theory for the relativistic transformation laws of Thermodynamics.

Some doubts about the validity of the Jüttner distribution function [19] have appeared due to the difficulty of formulating a relativistically consistent Hamiltonian approach for the interacting particles case [20]. Nevertheless, recently, Cubero et al. [21] have claimed that by using a bidimensional simulation, they reproduced the Jüttner distribution function in a moving system [Js] in one dimension. Albeit a consistent Hamiltonian formulation apparently cannot be reached for interacting particles [20,22], the Js is obtained just for a relativistic ideal gas and consequently the result is theoretically correct. Moreover, its validity is reinforced by the recent work done by Debbasch [23]. By doing a critical review of all arguments in favor of both the Js and the alternative distribution, Debbasch [23] arrived at the conclusion that the Js is correct and that the alternative one is not. Dunkel et al. $[24,25]$ derived two distributions based on different underlying reference measures. Even though, the above approach clarifies the mathematical differences on a fundamental level, it does not permit a decision to be made as to which distribution is actually the better candidate, as either reference measure has its own merits. Another important covariant approach has been done by Lehmann [26] which differs from Jüttner result. In fact, in Lehmann's paper, a many particle system is described in a Hamiltonian formulation with a canonical representation of the Poincare group but the world lines are considered invariant as an Anzatz (World line condition). Recently, Requardt [27] has made a profound analysis of the relativistic thermodynamics. Among others ideas, he gave a key role to the Lorentz Transformations in order to construct a relativistic Carnot cycle which allows to define a relativistic transformation of the temperature. Therefore, he also contributed to demonstrate that Landsberg claim [11] about the impossibility of defining a relativistic transformation law of the temperature was unfounded. As we mentioned before, Ott's proposal [5] represents one of the more important paper in the field such that Möller [28] changed his point of view about Relativistic Thermodynamics in the second edition of his book of Relativity switching to the Ott version instead of the PE proposal. Based somewhat on Möller point of view, Requardt developed a covariant proposal which is able to show many gaps and inconsistencies of Ott theory and Rohrlich theory. Moreover, by using the second and first laws of Thermodynamics and by defining some 4-vectors related with thermodynamical quantities, Requardt gave a detailed analysis of the so-called zeroth law of relativistic thermodynamics. However, even for 
many authors by correcting their inconsistencies, the Ott-Möller proposal represents the best theory which describes the relativistic transformations of thermodynamical quantities, it has been showed by Ares de Parga and López-Carrera [18] that different covariant approaches can be derived. A particular approach is obtained by choosing a volume in a reference frame. The PE approach represents one of these different proposals. As we will see later in the paper, the Rohrlich-Gamba proposal is another one and it possesses many similitudes with the ideas of Ott, Möller and Requardt. By using the RRT and a statistical theory developed by López-Carrera and Ares de Parga [13] based on the Balescu Statistical Mechanics theory $[6,12,13]$, they [13] have obtained the relativistic Js of an ideal gas. Nevertheless, the result is based on a non-covariant theory. As we mentioned before, Ares de Parga and López-Carrera [18], by using Nakamura's method [14,29], found a covariant theory of Thermodynamics but the corresponding relativistic covariant statistical theory has not already been developed. One of the purpose of this article consists of obtaining the Js [3] in a covariant form.

On the other hand, since nowadays some proposals have appeared formulating a relativistic consistent Hamiltonian approach to the interacting particles case [30], a discussion between Relativistic Thermodynamics and a possible statistical model for the interacting case will be exposed. Indeed, by disregarding the non-interacting theorem [20], a Statistical Relativistic Mechanics could be developed based on the covariant validity of the RRT. This represents the main purpose of the article. In order to describe the proposal, we will be obligated to describe the RRT and its relation with non-interacting Relativistic Statistical Mechanics showing for example a covariant deduction of the Js.

The article is organized as follows: in Section 2, we shortly describe a covariant theory of Relativistic Thermodynamics (RRT) and we analyze two important approaches. A covariant statistical theory for the non-interacting case is presented in Section 3 leading to a covariant correct form for obtaining the Js. In Section 4, a discussion about generalizing the model to the interacting case is presented. Some concluding remarks are exposed in Section 5.

\section{The Covariant Redefined Relativistic Thermodynamics}

\subsection{The General Case}

A relativistic theory must be accompanied by a covariant model. Some proposals have appeared in the literature claiming a covariant form of the Einstein-Planck proposal, as for example, Staruszkiewicz's proposal [31], among others. Nevertheless, those kinds of models are based on a false assumption; that is: it is assumed that the Einstein-Planck proposal conserves the invariance of the form of equilibrium Thermodynamics which has been proved to be incorrect by Ares de Parga et al. [12]. An interesting covariant theory was developed by van Kampen [15] leading to a scalar theory of the entropy, the temperature and the heat which contains similitudes with the different Rohrlich proposals [32]. Van Kampen model [15] which has been improved by Israel [16,17] and Nakamura [14,29], represents an interesting theory. By redefining some aspects of the last theory, Ares de Parga and López-Carrera [18] have proposed a covariant model which solves some of the inconsistencies of the van Kampen proposal [15]. In fact, Ares de Parga et al. [12] developed the so-called Redefined Relativistic Thermodynamics [RTT] and due to the fact of not being covariant some changes have been incorporated to it [18]. A covariant theory must have well-defined quantities when finite size systems are considered 
and different inertial systems are involved as the volume and any quantity derived by an integration over a volume of a vector or a tensor, as the total momentum. Fermi [33], Synge [34], Rohrlich [35], Gamba [36] and Nakamura [14] have showed the correct form of defining those physical quantities without ambiguities.

Let us present a summary of the revisited RTT. Consider a bar of length $l_{o}$ at rest in a reference frame $K_{o}$ (the subscript " $"$ means that the quantity is measured in the reference frame where the system is at rest). In another reference frame $K$ with relative velocity $u$ with respect the frame $K_{o}$, in the X-axis along the bar, we measure the length $l$ of the bar; that is: we take the two events in $K$, described by

$$
A_{1}^{\mu}=(0,0) \quad \text { and } \quad A_{2}^{\mu}=(0, l)
$$

where we have considered simultaneity between the two events. In $K_{o}$, the two events are described by

$$
A_{o 1}^{\mu}=(0,0) \quad \text { and } \quad A_{o 2}^{\mu}=\left(\gamma \frac{u}{c} l, \gamma l\right)
$$

It has to be noticed that the events in $K_{o}$ are not simultaneous but due to the fact that the bar is at rest in $K_{o}$, we can assure that

$$
\gamma l=l_{o}
$$

That is, Lorentz contraction is obtained. Moreover, the bar in $K$ has been represented by a set $A$ of events $A^{\mu}$ in $K$ such that

$$
A^{\mu}=(0, x) \quad \text { with } \quad x \epsilon[0, l] \quad \text { where } \quad l=l_{o} / \gamma
$$

This set $A$ in $K_{o}$ is described by all the events $A_{o}^{\mu}$ such that

$$
A_{o}^{\mu}=\left(\gamma \frac{u}{c} x, \gamma x\right) \text { with } x \epsilon[0, l]
$$

On the other hand, the regular bar in $K_{o}$ is represented by a set $B$ of events $B_{o}^{\mu}$ in $K_{o}$ such that

$$
B_{o}^{\mu}=(0, x) \quad \text { with } \quad x \epsilon\left[0, l_{o}\right]
$$

The conclusion is that set $A$ which represents the bar in $K$ does not correspond to the set $B$ which represents the bar at rest in $K_{o}$. Therefore, we can conclude that when we consider a volume, we are obligated to describe the set of events which defines the volume. Then, if we want to consider a volume by using a body we need to define, first of all, the reference frame where we will deal with the volume and secondly, the frame where the body is at rest. Mathematically, as Nakamura [14] proposed, we can describe the volume as follows: a three dimensional flat plane is defined as a set of events that satisfies $w^{\mu} x_{\mu}=0$ where $w^{\mu}$ represents the time-like unit vector that defines the direction of the three-dimensional volume in the four-dimensional space; that is, $w^{\mu}$ describes the volume. The volume is defined as the intersection of this flat plane and the world tube of the object. If we consider a frame $K$, we need to use the unit vector $u^{\mu}$ which represents the motion between $K$ and $K_{o}$ ( $u^{\mu}$ describes the observer frame $K)$. Then, the volume $V_{K}(w)$ is defined as

$$
V_{K}(w)=\frac{V_{o}}{u^{\lambda} w_{\lambda}}
$$


and the corresponding $4-$ vector volume

$$
V_{K}^{\mu}(w)=\frac{w^{\mu} V_{o}}{u^{\lambda} w_{\lambda}}
$$

For examples, let us analyze two different cases (let us permit to work in one dimension and let us call a 2 -vector as a 4 -vector).

Case 1 or Rohrlich-Gamba [RG] proposal

If we want to deal with the volume which represents all the events that simultaneously occur in the rest frame and if we look this volume from the frame $K$, we have

$$
w^{\mu}=\left(\gamma, \gamma \frac{u}{c}\right) \quad \text { and } \quad u^{\mu}=\left(\gamma, \gamma \frac{u}{c}\right)
$$

That is $w^{\mu}=u^{\mu}$. Consequently,

$$
u^{\lambda} w_{\lambda}=1
$$

The considered volume is

$$
V_{K_{o}}(w)=\frac{V_{o}}{u^{\lambda} w_{\lambda}}=V_{o}
$$

and the corresponding $4-$ vector volume is represented by

$$
V_{K_{o}}^{\mu}(w)=\frac{w^{\mu} V_{o}}{u^{\lambda} w_{\lambda}}=w^{\mu} V_{o}=u^{\mu} V_{o}=\left(\gamma, \gamma \frac{u}{c}\right) V_{o}
$$

Therefore, in this case, the differential of the volume is:

$$
d V_{K_{o}}^{\mu}(w)=\frac{w^{\mu} d V_{o}}{u^{\lambda} w_{\lambda}}=w^{\mu} d V_{o}=\left(\gamma, \gamma \frac{u}{c}\right) d V_{o}
$$

which represents the 4-vector differential of the volume that Gamba [36] has used in order to clarify the electromagnetic controversy of the $(4 / 3)$ term.

Case 2 or Planck-Einstein [PE] proposal

Let us now consider a volume generated by a Lorentz contraction in $K$ of a body whose volume $V_{o}$ is at rest in $K_{o}$. We know that this volume is equal to $V_{K}=\gamma^{-1} V_{o}$ in $K$. Let us check it. As we noticed before, all the points of this volume are simultaneous in $K$ and, consequently, we can consider it as a volume at rest in $K$. In the Nakamura formalism, the different $4-$ vectors will be described in $K$ by

$$
w^{\mu}=(1,0) \quad \text { and } \quad u^{\mu}=\left(\gamma, \gamma \frac{u}{c}\right)
$$

Consequently,

$$
u^{\lambda} w_{\lambda}=\gamma
$$

Now, our volume is defined as:

$$
V_{K}(w)=\frac{V_{o}}{u^{\lambda} w_{\lambda}}=\frac{V_{o}}{\gamma}
$$

and the $4-$ vector volume from $K$ is

$$
V_{K}^{\mu}(w)=w^{\mu} \frac{V_{o}}{u^{\lambda} w_{\lambda}}=w^{\mu} \frac{V_{o}}{\gamma}=(1,0) \frac{V_{o}}{\gamma}
$$


Therefore, in order to avoid confusion, it is convenient to define the volume by giving the value of the $4-$ vector $w^{\mu}$ and $u^{\mu}$ in the frame $K$.

Since the volume has been well-defined by applying this method, we assure that we will obtain well-defined quantities at the time of integrating any vector $F^{\mu}$ or tensor $F^{\mu \nu}$ over the volume; that is,

$$
\Gamma=\int_{V_{K}\left(w^{\mu}\right)} F^{\mu} d V_{\mu} \quad \text { or } \quad \Gamma^{\mu}=\int_{V_{K}\left(w^{\mu}\right)} F^{\mu \nu} d V_{v}
$$

represent a well-defined invariant and a 4 -vector, respectively.

Let us next calculate the momentum of a system. First of all, we have to specify which is the reference frame where we define the volume: that is, we give the value of $w^{\mu}$ in a reference frame $K$. The energy-momentum density tensor [37] $T^{\mu \nu}$ is supposed to be constant within the volume when the system is in thermal equilibrium. Therefore, we can define the 4 -vector momentum as

$$
P^{\mu}(w)=\int_{V_{K}\left(w^{\mu}\right)} T^{\mu v} d V_{\nu}=\frac{V_{o} T^{\mu \nu} w_{\nu}}{u^{\lambda} w_{\lambda}}=T^{\mu \nu} V_{\nu}
$$

Once we have a covariant expression for the 4-momentum (energy-momentum), following van Kampen [15] and Nakamura [14], the entropy change of the object is

$$
d S=\beta_{\mu} \frac{V_{o} w_{\nu}}{u^{\lambda} w_{\lambda}} d T^{\mu \nu}+\beta_{\mu} \mathbb{P} d V^{\mu}
$$

where $\beta_{\mu}=\frac{u_{\mu}}{k T_{o}}$, known as the 4-inverse temperature [14-17]. Equation (14) represents the main contribution of Nakamura [14] to the van Kampen theory [15]. The energy-momentum density tensor is defined by

$$
T^{\mu \nu}=\left(\mathbb{P}+e_{o}\right) u^{\mu} u^{v}-\mathbb{P} g^{\mu \nu}
$$

where $e_{o}$ represents the energy density in the rest frame and $\mathbb{P}$ the pressure. $T^{\mu \nu}$ coincides with the regular stress tensor described by Weinberg [38] or Landau and Lifshitz [37]. Let us now understand the meaning of Equation (14) by using Equation (15). Since the entropy is defined as a contraction of a tensor, it represents an invariant. That is:

$$
\begin{aligned}
d S & =\beta_{\mu} \frac{V_{o} w_{\nu}}{u^{\lambda} w_{\lambda}} d T^{\mu \nu}+\beta_{\mu} \mathbb{P} d V^{\mu} \\
& =\beta_{\mu} \frac{V_{o} w_{\nu}}{u^{\lambda} w_{\lambda}} d\left(\left(\mathbb{P}+e_{o}\right) u^{\mu} u^{v}-\mathbb{P} g^{\mu \nu}\right)+\beta_{\mu} \mathbb{P} d V^{\mu} \\
& =\frac{u_{\mu}}{k T_{o}} \frac{V_{o} w_{\nu}}{u^{\lambda} w_{\lambda}} d\left(\left(\mathbb{P}+e_{o}\right) u^{\mu} u^{v}-\mathbb{P} g^{\mu \nu}\right)+\frac{u_{\mu}}{k T_{o}} \mathbb{P} \frac{w^{\mu} d V_{o}}{u^{\lambda} w_{\lambda}} \\
& =\frac{1}{k T_{o}} \frac{V_{o}}{u^{\lambda} w_{\lambda}} d\left(\left(\mathbb{P}+e_{o}\right) w_{\nu} u^{v}-\mathbb{P} w_{\nu} u^{\nu}\right)+\frac{u_{\mu}}{k T_{o}} \mathbb{P} \frac{w^{\mu} d V_{o}}{u^{\lambda} w_{\lambda}} \\
& =\frac{1}{k T_{o}} d E_{o}+\frac{\mathbb{P}}{k T_{o}} d V_{o}
\end{aligned}
$$

where we have defined $E_{o}=e_{o} V_{o}$. The result is obtained without specifying which kind of volume. The reason lies in the fact that the entropy is related with the number of states which is independent of the choice of the volume. Of course, this is due to the fact of dealing with a stationary system. 
Let us now calculate the momentum:

$$
\begin{aligned}
P^{\mu}(w) & =\left(\left(\mathbb{P}+e_{o}\right) u^{\mu} u^{\nu}-\mathbb{P} g^{\mu \nu}\right) \frac{V_{o} w_{\nu}}{u^{\lambda} w_{\lambda}} \\
& =V_{o}\left(\left(\mathbb{P}+e_{o}\right) u^{\mu}-\mathbb{P} \frac{w^{\mu}}{u^{\lambda} w_{\lambda}}\right) \\
& =\left(\mathbb{P} V_{o}+E_{o}\right) u^{\mu}-\mathbb{P} V_{o} \frac{w^{\mu}}{u^{\lambda} w_{\lambda}}
\end{aligned}
$$

The momentum of the system will depend on the choice of the volume. Nevertheless, If we want to make a correspondence with the first law,

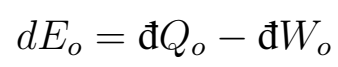

we need to generalize Equation (18) for any choice of the volume and reference frame. It is necessary to define the redefined energy-momentum. First of all, we must define the 4 -vector $G^{\mu}$ as

$$
\begin{aligned}
G^{\mu} & =\left(\left(\mathbb{P}+e_{o}\right) u^{\mu} u^{\nu}-\left(\mathbb{P}+e_{o}\right) g^{\mu \nu}\right) \frac{V_{o} w_{\nu}}{u^{\lambda} w_{\lambda}} \\
& =\left(\mathbb{P} V_{o}+E_{o}\right) u^{\mu}-\left(\mathbb{P} V_{o}+E_{o}\right) \frac{w^{\mu}}{u^{\lambda} w_{\lambda}} \\
& =\left(\mathbb{P} V_{o}+E_{o}\right)\left(u^{\mu}-\frac{w^{\mu}}{u^{\lambda} w_{\lambda}}\right)
\end{aligned}
$$

and then we construct the $4-$ vector redefined energy-momentum, $\xi^{\mu}$ :

$$
\begin{aligned}
\xi^{\mu} & =P^{\mu}-G^{\mu} \\
& =\frac{w^{\mu}}{u^{\lambda} w_{\lambda}} E_{o}
\end{aligned}
$$

Notice that in the RG case, $G^{\mu}$ vanishes, that is,

$$
\begin{aligned}
G_{R G}^{\mu} & =\left(\mathbb{P} V_{o}+E_{o}\right)\left(u_{R G}^{\mu}-\frac{w_{R G}^{\mu}}{u_{R G}^{\lambda} w_{R G \lambda}}\right) \\
& =\left(\mathbb{P} V_{o}+E_{o}\right)\left(u_{R G}^{\mu}-\frac{u_{R G}^{\mu}}{1}\right)=(0,0)
\end{aligned}
$$

As a consequence, we have $P_{R G}^{\mu}=\xi_{R G}^{\mu}$.

Therefore, we can assure that for any chosen volume, a covariant relativistic Thermodynamics, the RRT, can be described by the following relations

$$
d \xi^{\mu}=đ \Theta^{\mu}-\mathrm{d} W^{\mu}
$$

where

$$
d \xi^{\mu}=w^{\mu} \frac{d E_{o}}{u^{\lambda} w_{\lambda}}, \quad \text { đ } \Theta^{\mu}=w^{\mu} \frac{\mathrm{đ} Q_{o}}{u^{\lambda} w_{\lambda}} \quad \text { and } \quad \text { đ } W^{\mu}=w^{\mu} \frac{\mathbb{P} d V_{o}}{u^{\lambda} w_{\lambda}}
$$

and

$$
T^{\mu}=w^{\mu} \frac{T_{o}}{u^{\lambda} w_{\lambda}} \quad \text { and } \quad \beta_{\mu}=\frac{u_{\mu}}{k T_{o}}
$$


with the entropy expressed as

$$
d S=\beta_{\mu} \frac{V_{o} w_{\nu}}{u^{\lambda} w_{\lambda}} d T^{\mu \nu}+\beta_{\mu} \mathbb{P} d V^{\mu}
$$

The thermodynamical relations are obtained just by defining the volume. The Ott-Rohrlich proposal is obtained by choosing the RG volume. A corrected Redefined Relativistic Thermodynamics, RRT, is also found by using the PE volume. The interesting fact is that for any other choice of the volume we will obtain the thermodynamical relations. It can be thought that this new theory does not contribute

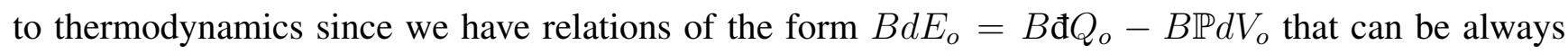
simplified to $d E_{o}=\mathrm{d} Q_{o}-\mathbb{P} d V_{o}$, that is, the Thermodynamics in the rest frame. Nevertheless, when two systems are considered, this formalism will be useful. This has been applied to the study of two blackbodies interacting with the same initial conditions but with different motions given a consistent result [18] which avoids the van Kampen's lack of conservation of heat.

\subsection{The Planck-Einstein Case and Its Thermodynamics}

Let us present, in a not covariant form, a special resume of the theory when the PE volume is chosen. The last formalism keeps the invariance of the form in Thermodynamics and by using the first component of the quantities of the theory developed above, we obtain the following quantities: the volume $V=V_{P E}=V^{0}=\gamma^{-1} V_{o}$, the redefined energy $\xi$ has been defined [12] as

$$
\xi=E-\gamma \frac{u^{2}}{c^{2}}\left(E_{o}+\frac{u^{2}}{c^{2}} \mathbb{P} V_{o}\right)
$$

where $E$ represents the relativistic PE transformed internal energy. We know that the energy transforms as

$$
E=\gamma\left(E_{o}+\frac{u^{2}}{c^{2}} \mathbb{P} V_{o}\right)
$$

Therefore,

$$
\xi=\gamma\left(E_{o}+\frac{u^{2}}{c^{2}} \mathbb{P} V_{o}\right)-\gamma\left(E_{o}+\mathbb{P} V_{o}\right) \frac{u^{2}}{c^{2}}=\gamma^{-1} E_{o}
$$

that is:

$$
\xi=\xi^{0}
$$

Therefore, the first component of the 4-vector redefined energy-momentum coincides with the redefined energy $[12,18]$. Moreover, By using Equation (17), we can notice that for the PE case

$$
\begin{aligned}
P & =\left(\mathbb{P} V_{o}+E_{o}\right) u^{\mu}-\mathbb{P} V_{o} \frac{w^{\mu}}{u^{\lambda} w_{\lambda}} \\
& =\left(\mathbb{P} V_{o}+E_{o}\right) \gamma \frac{u}{c}
\end{aligned}
$$

Therefore, by using Equation (26), it is easy to see that

$$
\xi=E-P \frac{u}{c}
$$

which will be of great use when we analyze Relativistic Statistical Mechanics. Table 1 describes the more important transformed quantities: ( $F$ represents the Helmholtz free energy) 
Table 1. Relativistic transformations for the PE case.

\begin{tabular}{ccccccc}
\hline$V$ & $\mathbb{P}$ & $S$ & $T$ & $đ \Theta$ & $\xi$ & $F$ \\
\hline$\frac{V_{o}}{\gamma}$ & $\mathbb{P}_{o}$ & $S_{o}$ & $\frac{T_{o}}{\gamma}$ & $\frac{đ}{\gamma} Q_{o}$ & $\frac{\xi_{o}}{\gamma}=\frac{E_{o}}{\gamma}$ & $\frac{F_{o}}{\gamma}$ \\
\hline
\end{tabular}

The difference with the "Renormalized" Relativistic Thermodynamics [12] consists of noticing that the heat has been redefined as the redefined heat and the work has kept its form without adding to it the bulk energy $\left(\gamma\left(E_{o}+\mathbb{P} V_{o}\right) \frac{u^{2}}{c^{2}}\right)$ [18]. The work transforms as $d W=\mathbb{P} \gamma^{-1} d V_{o}$. With this definition, we obtain all the thermodynamical relations, as for example, the first law, that is,

$$
d \xi=đ \Theta-\mathbb{P} d V
$$

\subsection{The Rohrlich-Gamba Case and Its Thermodynamics}

Let us now present, in a not covariant form, a special resume of the theory when the RG volume is considered.

$V=V_{R G}=V^{0}=\gamma V_{o}$, the redefined energy $\xi=\xi^{0}$

$$
\xi=\xi^{0}=\gamma E_{o}
$$

Table 2 describes the more important transformed quantities: ( $F$ represents the Helmholtz free energy)

Table 2. Relativistic transformations for the RG case.

\begin{tabular}{ccccccc}
\hline$V$ & $\mathbb{P}$ & $S$ & $T$ & $đ \Theta$ & $\xi$ & $F$ \\
\hline$\gamma V_{o}$ & $\mathbb{P}_{o}$ & $S_{o}$ & $\gamma T_{o}$ & $\gamma ₫ Q_{o}$ & $\gamma E_{o}$ & $\gamma F_{o}$ \\
\hline
\end{tabular}

The differences between the PE case and the RG case is that the bulk energy vanishes in this case $\mathrm{RG}$ and a $\gamma$ factor appears instead of the $\gamma^{-1}$. We have to notice that Table 2 describes exactly the second Rohrlich's proposal [32]. With this definition, we obtain all the thermodynamical relations, as for example, the first law, Equations (18) and (32) which has also been obtained in the PE case. We have to notice that the first component of the $4-$ vector $T^{\mu}$ is $T^{0}=\gamma T_{o}$ but $\beta_{\mu}=u_{\mu} / k T_{o}$.

We have to remember, as we noticed at the end of Section 2.1, that the formalism is equivalent to the regular Thermodynamics when the systems are in the same rest frame, but the application emerges when two different systems are considered and their rest frames are different as it has been applied in the case of two moving blackbodies [18].

It has to be noticed that the results described in Tables I and II show and correct the gauge theory developed by Balescu [6]. Now that we exposed the RRT, we can then develop a covariant statistical theory.

\section{Covariant Relativistic Statistical Mechanics}

Let us start by considering that the distribution function [6],

$$
f_{o}=\alpha \exp -\frac{H(q, p)}{k T_{o}}, \quad \text { with } \quad \alpha=\frac{1}{Z}
$$


satisfactorily describes the Relativistic Statistical Mechanics of a system which total momentum vanishes for a non-interacting system. However, since the RRT is independent of the interaction of the system, we can suspect that a canonical transformation could be derived in order to obtain the RRT. Nevertheless, the no-interaction theorem [20] does not allow such a transformation to be used for the general case of an interacting system. This will be analyzed in Section 4 by using the RRT. By the time, we will just try to obtain a covariant Relativistic Statistical Mechanics for non-interacting systems consistent with the RRT. We are tempted to start with the relativistic distribution function which describes the system at rest, Equation (34) and to make a change of variable in order to obtain the distribution function in another reference frame. Nevertheless, a change of variable, that is a Lorentz transformation between the coordinates in the rest frame $K_{o}$ and the coordinates in the moving frame $K$, would not have any sense, as we will explain later on the paper, due to the non-simultaneity of the Lorentz transformation [39]. However in some situations, a change of variable as the transformation of the momentum in the moving frame to the rest frame will bring us to reasonable results but as we will see, it is just a mere coincidence due to the type of involved integrals. Consequently, canonical transformations must be used [6] in order to obtain the distribution function in a moving reference frame. However, this will be possible just for the no-interacting case [20] by using the regular notion of simultaneity. Even in the non-interacting case, we must be careful not to confuse the canonical transformations with changes of variables.

On the other hand, it has to be noticed that even if Balescu [6] has committed the mistake of considering the invariance of the regular relativistic thermodynamics [12,13,18], his proposal for a Relativistic Statistical Mechanics is correct under the assumption that the Thermodynamics deduced from it is the RRT. In order to explicitly shows this aspect, this connection has to be done by using the covariant representation of the RRT. The better example showing the goodness of the theory will be presented when the Js will be obtained.

It has to be pointed out that the RG case will not be analyzed anymore because of the experimental lack of application. Normally we are just interested in measuring quantities in our laboratory frame. Therefore, we will work from now until the end of the article with the PE case.

\subsection{MeanIngless of the Change of Variable}

In order to understanding the aim of this subsection, it is necessary to present some background. In fact, the number of attempts to develop a Relativistic Statistical Mechanics is endless. However, among others, there are two important lines of thought represented by the works of Hakim [40] and Balescu [6] which are somewhat divergent at some points. From Lorentz transformations and by constructing 4-current vectors, Hakim [40] proposal found distributions functions which satisfy a Liouville type equation. Moreover, this formalism allows him to develop a Relativistic Statistical Mechanics of charged particles given a modification of the Vlasov equation [41] (Unbelievably, this work has not been cited as it deserves.). On the other hand, with awareness of the no-interaction theorem [20], Balescu developed a statistical mechanics for non-interacting particles and in equilibrium based on the theory of canonical transformations. However, the lack of covariance in his formalism led him to make a mistake. In fact, the PE proposal does not conserve the invariance of the form as Ares de Parga et al. [12] have noticed. This justifies Hakim sentence [40] about Balescu work: "However, besides a number of conceptual difficulties (both mathematical and physical) the transformation properties of these theories are not yet 
completely clear and we no longer consider them in this paper". Nevertheless, due to the no-interaction theorem, Balescu [6] abandoned the idea of analyzing the time evolution of a system. This constraints, imposed by Balescu along with Hakim sentence "... there is no canonical notion of evolution of a relativistic system of particles", showed the differences between the purposes of the two points of view. However, within the Balescu philosophy, there are two points that has to be corrected in order to obtain a consistent PE theory. One of them consists of dealing with well-defined 4-vectors which has been explained in Section 2.1 and it has justified the use of the RRT. The other point is to emphasize the correct use of canonical transformations in order to also obtain well-defined quantities in Relativistic Statistical Mechanics theory which will be described in Section 3.2 while in this subsection we pretend to explain a common confusion about the lack of sense of the change of variable within the theory.

Let us begin by describing the Landau and Lifshitz [42] point of view. Arguing geometrical reason, they proved the invariance of

$$
\frac{d p^{\prime}}{E^{\prime}}=\frac{d p}{E}
$$

where $p, E$ and the superscript " '" represent the momentum, the energy and the representation of the quantity in the $K^{\prime}$ frame, respectively. This result can be also obtained by integrating a delta function $\delta\left(p^{\mu} p_{\mu}-m^{2} c^{2}\right)$ for a $d p^{4}$ integration. Let us consider the following invariant integral

$$
\begin{aligned}
\int \theta\left(p_{0}\right) \delta\left(p^{\mu} p_{\mu}-m^{2} c^{2}\right) d p^{0} d p^{1} d p^{2} d p^{3} & =\int \theta\left(p_{0}\right) \frac{1}{c} \delta\left(p_{0}^{2}-\frac{E^{2}}{c^{2}}\right) d p^{4} \\
& =\int \frac{d p_{x} d p_{y} d p_{z}}{E}
\end{aligned}
$$

where $\theta\left(p_{0}\right)$ represents the Heaviside function. Due to the invariant property of the last integral, Equation (35) is justified. However without making any formal definition (remember that we are just describing Landau and Lifshitz proposal), Landau and Lifshitz introduced what they called a distribution function such that the integration over $d p$ represents the number of particles; that is:

$$
\int F d p=N=\int F^{\prime} d p^{\prime}
$$

where $N$ represents the number of particles of the system which is an invariant. However, once $F$ is integrated over $d p$ like in Equation (36), it becomes space density and it must represent the 0-component of a 4 -vector which satisfies the continuity equation. Nevertheless, this can be clarified by considering that if they would have defined the one-component distribution function $f$, such that

$$
\int f d p d V=N
$$

the integration of $f$ over $d p$,

$$
\int f d p
$$

would represent a space density which possesses the property of a 0 -component of a space density. Therefore, for independence of the position case, we have that

$$
F=\int f d V=f V
$$


Since the volume transforms $V=\gamma^{-1} V_{o}$, it is evident that $\int f d p$ will behaves like the 0 -component of a 4-vector space density. It has to be remembered that integrals over a volume may not be well-defined. Therefore, the technique described in Section 2.1 must be applied in order to well-define the integral.

Therefore,

$$
\int F \frac{E}{E} d p=\int F^{\prime} \frac{E^{\prime}}{E^{\prime}} d p^{\prime}
$$

By using Equation (35), we arrive at

$$
F E=F^{\prime} E^{\prime}
$$

Or in a better form,

$$
F^{\prime}=\frac{E^{\prime}}{E} F
$$

Nevertheless, this result is just a disguised change of variable. Let us consider the transformation of the momentum from a $K^{\prime}$ frame which moves with a velocity $u$ with respect to a $K$ frame:

$$
p^{\prime}=\gamma\left(p-\frac{u}{c^{2}} E\right) \quad \text { and } \quad E^{\prime}=\gamma(E-u p)
$$

Since

$$
E=\sqrt{p^{2} c^{2}+m^{2} c^{4}}
$$

we obtain

$$
\begin{aligned}
& F^{\prime} d p^{\prime}=F^{\prime}\left(p^{\prime}(p)\right) \frac{d p^{\prime}}{d p} d p \\
& F^{\prime} d p^{\prime}=F^{\prime}\left(p^{\prime}(p)\right) \gamma\left(1-u \frac{p}{E}\right) d p
\end{aligned}
$$

That is, by using Equation (40), we have

$$
\begin{aligned}
\int F^{\prime} d p^{\prime} & =\int F^{\prime}\left(p^{\prime}(p)\right) \gamma\left(1-u \frac{p}{E}\right) d p \\
& =\int F^{\prime}\left(p^{\prime}(p)\right) \frac{\gamma}{E}(E-u p) d p \\
& =\int \frac{E^{\prime}}{E} f^{\prime}\left(p^{\prime}(p)\right) d p
\end{aligned}
$$

We arrive to the same result obtained by Landau and Lifshitz [42]. Finally, we can conclude that Landau and Lifshitz method is equivalent to a change of variable. Nevertheless, in both cases, taking into account Equation (36), we cannot conclude that

$$
\frac{E^{\prime}}{E} F^{\prime}\left(p^{\prime}(p)\right)=F(p)
$$

Equations (39) and (43) does not imply the identity expressed in Equation (44). It just assure that

$$
\int F^{\prime} d p^{\prime}=\int \frac{E^{\prime}}{E} F^{\prime}\left(p^{\prime}(p)\right) d p=\int F^{\prime}\left(p^{\prime}(p)\right) \gamma\left(1-u \frac{p}{E}\right) d p
$$

Moreover, the function $F^{\prime}\left(p^{\prime}(p)\right)$ just represent the distribution function measured in the reference frame $K^{\prime}$ by using as a parameter the corresponding momentum in the reference frame $K$. It cannot be related with the distribution function $F(p)$ in $K$. As we noticed above the role of the volume has not been taken 
into account and an extra term $\gamma^{-1}$ must appear when change of variable are done passing from $\mathrm{K}^{\prime}$ to K. Normally the distribution function is an even function on $p$, therefore, Equation (45) is easy to be calculated, that is:

$$
\int F^{\prime} d p^{\prime}=\int F^{\prime}\left(p^{\prime}(p)\right) \gamma\left(1-u \frac{p}{E}\right) d p=\int F^{\prime}\left(p^{\prime}(p)\right) \gamma d p
$$

where $\gamma$ would disappear when the integration over the volume is done. That is

$$
\int f^{\prime} d p^{\prime}=\int f^{\prime}\left(p^{\prime}(p)\right) d p
$$

A very similar mistake has been done by Van Kampen [43]. By using the distribution canonical function $f(q, p)$, Balescu [6] used in a similar way Equation (46) in order to demonstrate the invariance of the partition function. But Balescu asseveration is true because in his model

$$
f^{\prime}\left(q^{\prime}(q), p^{\prime}(p)\right)=f(q, p)
$$

and the integration of the term containing $-u \frac{p}{E}$ vanishes. Surprisingly, Balescu [6] used a change of variable to calculate the invariance of the partition function. His model is derived by the correct idea of using canonical transformation and the result is obtained due to the even character of the distribution function. That is: it is a mere coincidence. The $\gamma$ term does not appear since dealing with the canonical distribution function the volume integral must be done. However, when we make a change of variable at the moment of calculating an observable, even if $g \neq g(q)$, we will have

$$
\langle g\rangle^{\prime}=\int g\left(p^{\prime}\right) f^{\prime}\left(p^{\prime}\right) d p^{\prime} d q^{\prime}=\int g\left(p^{\prime}(p)\right) f^{\prime}\left(p^{\prime}(p)\right) \gamma\left(1-u \frac{p}{E}\right) d q d p
$$

Even if we substitute $f^{\prime}\left(p^{\prime}(p)\right)$ by $\left.f(p)\right)$, in general, the term $-u \frac{p}{E}$ in the Jacobian $d p^{\prime} / d p=\gamma\left(1-u \frac{p}{E}\right)$ would play an important role. This implies that the corresponding observable $\langle g\rangle$ will not be related with $\langle g\rangle^{\prime}$ in a natural way. Another example of the meaningless of using a change of variable is described when the transformation of the Js, $f_{J s}$, is obtained by using Equation (39), the result will be related with the Jüttner, $f_{J}$, distribution function by

$$
f_{J s}^{\prime}\left(p^{\prime}\right)=\frac{1}{\left(1-u \frac{p}{E}\right)} f_{J}(p)
$$

which is not good enough as we will see later on the paper. Moreover, if an expression as Equation (48) is used and the Js is obtained with the help of $\delta\left(p_{i}-p\right)$, the result will be exact. This represent another time, as in the case of the partition function calculated by Balescu [6], a mere coincidence. The reason is related with the point character of the delta function where the Jacobian coincides with the denominator factor that appears when a change of variable is done.

Therefore, we can conclude that if we want to calculate any kind of distribution function in a moving frame, a change of variable will not represent a serious method to obtain the result. That is when a change of variable is used and the result is accurate, it is just a mere coincidence. The reason is the following: when we are trying to describe the canonical distribution function in a particular frame, it has to be considered that each position is measured at the same time. But a Lorentz transformation implies a non-simultaneity for each event. 
This problem has been analyzed by many authors, in particular by Balescu and Kotera [39] and surprising Balescu [6] committed a mistake in his classical article. Indeed, $f^{\prime}\left(p^{\prime}(p)\right)$ represents the distribution function at $K^{\prime}$ as a function of a parameter $p$ defined by Equations (40) and (41) and not the distribution function $f(p)$ at $K$ as we noticed before.

Intuitively, we can correct this fact by saying that the distribution function must be calculated at a time. Since when the Lorentz transformation is considered a non-simultaneity appears and in order to connect both distribution functions, the transformation of the momentum has to be corrected by the factor which gives an extra momentum do the interval of time $d t$ appeared due to the proper time of the rest frame; that is:

$$
p^{\prime}=\gamma\left(p-\frac{u}{c^{2}} E+\Delta P\right) \quad \text { with } \quad \Delta P=m u \frac{d t}{d \tau}
$$

That is,

$$
\Delta P=m u \frac{d t}{d \tau}=\frac{u}{c^{2}} m c^{2} \gamma=\frac{u}{c^{2}} E
$$

Therefore, the transformation must be taken as

$$
p^{\prime}=\gamma\left(p-\frac{u}{c^{2}} E+\frac{u}{c^{2}} E\right)=\gamma p
$$

Finally,

$$
d p^{\prime}=\gamma d p
$$

Therefore, this intuitive transformation will give for the $n$-particle case,

$$
d q^{\prime} d p^{\prime}=\left(\gamma^{-1}\right)^{n} d q(\gamma)^{n} d p=d q d p
$$

which preserves the phase space volume as it is required in a canonical transformation. Equation (54) represents an intuitive result which cannot be used due to the same arguments that we present in Section 2.1 about the problem of simultaneity in relativity. However, due to the formalism presented in Section 2.1, it will be possible to give a covariant definition of the volume and the expression would be well-defined as we will see in Section 3.3 (Equations (98) and (99)). The purpose of this subsection was two fold: firstly, we demonstrate the meaningless of the change of variable; secondly, the necessity of introducing canonical transformations in the theory in order to be able to understand the integrals of the type $\int f d q d p$ which will be explained in the next subsections.

\subsection{Canonical Transformations}

In the introduction section, we notice that a consistent Hamiltonian formulation apparently cannot be reached for interacting particles [20]. Although the theory of canonical transformation has been developed many years ago, the work done by Curie et al. [20] represents a watershed moment. They showed that a boost canonical transformation cannot be used in order to obtain the transformation of a distribution function from a reference frame to another when a system of interacting particles is used. Nevertheless, if we consider a system of non-interacting particles, since for this case the center of motion problem is solved, a boost canonical transformation can be used in order to obtain the distribution function of a system of non-interacting particles. In fact, Balescu [6] has successfully used a boost canonical transformation in order to obtain the distribution function of a system of 
non-interacting particles. Moreover, he obtained a Relativistic Statistical Mechanics which generated a Relativistic Thermodynamics. Although he committed the mistake of misunderstanding the invariance of the laws of thermodynamics [12,13,18], by defining the redefined energy, his Relativistic Statistical Mechanics generates the RRT for the PE case. Let us make a resume of the theory of boost canonical transformations. If a system is at rest in a reference frame $K_{o}$, a distribution function $f(q, p, 0)$ represents the probability of finding the state of the system at $q$ and $p$ where $q$ represent the $n$ coordinates of the particles and $p$ the corresponding $n$ momentums. For example, a system of $n$ particles which coordinates are

$$
\left(x_{1}, y_{1}, z_{1}, \ldots, x_{n}, y_{n}, z_{n} ; p_{x 1}, p_{y 1}, p_{z 1}, \ldots, p_{x n}, p_{y n}, p_{z n}\right)
$$

is represented by $(q, p)$ and the volumes $d V_{1} d V_{2} \ldots d V_{n}$ and $d P_{1} d P_{2} \ldots d P_{n}$ are represented by $d q d p$. Therefore, by using Equation (34), we have,

$$
1=\int f_{o} d q_{o} d p_{o}=\int f(q, p, 0) d q_{o} d p_{o}
$$

where the subscript " $o$ " denotes that the phase space is considered in the rest frame $K_{o}$. An observable $\langle g\rangle_{o}$ is obtained by

$$
\langle g\rangle_{o}=\int g f(q, p, 0) d q_{o} d p_{o}
$$

This last equation represents the golden rule of Statistical Mechanics. When we are interested in observing a system from a reference frame which is not the rest frame, we are tempted to use the theory of canonical transformation. In fact, in a Hamiltonian formalism all physical laws are expressed by Poisson Bracket relations. Hence, the dynamical variables in a reference frame must be related to the expression in the rest frame by a transformation which leaves the Poisson bracket invariant; that is: by a canonical transformation. For each Lorentz transformation we shall associate a canonical transformation. The theory of Lie groups supply the rules for obtaining such transformations. Nevertheless, Curie et al. [20] have showed that for a system of interacting particles, it is impossible to obtain such canonical transformations. However, such transformation can be calculated for a system of non-interacting particles. For an equilibrium system with non-interacting particles, the distribution function will be independent of the time, $f \neq f(t)$; that is: $f=f(q, p)$. The distribution function in another reference frame will be described by $f^{\prime}\left(q^{\prime}, p^{\prime}\right)$. As we noticed in Section 2.1, in relativity we deal with 4 -vectors such that two simultaneous events in a reference frame will not be simultaneous in another frame. Consequently, we may think that in the new reference frame the distribution function will possesses a time dependence, $f^{\prime}=f^{\prime}\left(t^{\prime}\right)$. However, when we deal with canonical transformations the $q^{\prime}$ s in a reference frame do not correspond to the Lorentz transformations of the $q$ 's in another frame. That is, we just look for the functionality of the distribution function. In fact, we will see in Section 3.3 that the relation between the differentials $d q d p$ will be related by a canonical factor and not by a Jacobian function. The theory of Lie group predicts that such a canonical transformation must be described by a particular generator. Let us present a simple form for obtaining such generators. Let us begin by describing the transformation of a dynamical variable, in this case the distribution function, $f(p, q)$, under an infinitesimal transformation [39]

$$
x^{0}=x^{0}+\delta s x^{1} \quad \text { and } \quad x^{1}=x^{1}+\delta s x^{0}
$$


The infinitesimal transformation of $f(q, p)$ is given by

$$
f(p, q ; s)=f(p, q)+[f(p, q), \mathcal{K}(p, q)] \delta s
$$

where $\mathcal{K}$ represents the boost generator. Therefore, for a finite transformation $\left(\tanh s=\frac{u}{c}\right)$

$$
\frac{\partial f(p, q ; s)}{\partial s}=[f(p, q ; s), \mathcal{K}(p, q)]
$$

By solving this equation, the theory of canonical transformation permits to calculate the distribution function $f(q, p, s)$ in a reference frame $K$ which moves in the direction of the X-axis with a velocity $u / c=\tanh s$ with respect the rest frame $K_{o}$. The result is

$$
f(q, p, s)=\exp (\mathcal{K} s) f(q, p, 0)
$$

where $\mathcal{K}$ represents the boost in the $\mathrm{X}$-axis. That is in the reference frame $K$, we also have

$$
1=\int f(q, p, s) d q_{s} d p_{s}
$$

where $d q_{s} d p_{s}$ represents the phase space in the reference frame $K$.

It is important to remember that we are dealing with a system of non-interacting particles in equilibrium. Hence, the distribution functions in each reference frame will not depend on the time.That is, fortunately we will not have to compare expressions as

$$
f(q, p, t) d q d p=f^{\prime \prime}\left(q^{\prime}, p^{\prime}, t^{\prime}\right) d q^{\prime} d p^{\prime}
$$

since it will have no sense. In fact, if time is involved, we will not be able to understand the meaning of the last equation. Two simultaneous events in a frame $K$ will not be simultaneous in another reference frame $K^{\prime}$ and the distribution function in $K^{\prime}$ will not be represented by the same time over the phase space and the integral over the phase space will not have any sense. However, for an equilibrium system the distribution function will not depend on the time and if we realize that the canonical transformation just describes the functionality of a dynamical variable, the controversy cited above disappears.

It has to be pointed out that when a canonical transformation is used the phase space seems to remain invariant in this formalism; that is:

$$
d q_{s} d p_{s}=d q_{o} d p_{o}
$$

This is due to the form presented in Equation (56) and it coincides with our intuitive deduction of it, Equation (54). However, this is a point that it is not well-defined due the non-simultaneity effect. In Section 3.3, we will explain that Equation (60) represents a real identity provided the formalism developed in Section 2.1 is used, that is, the RRT. As an example of this, we can analyze the case of a one particle phase space: since we are dealing just with the PE case, we know that the volume $d V_{o}$ transforms to

$$
d V_{s}=\gamma^{-1} d V_{o}
$$

Consequently, if we want an identity similar to Equation (60), we will need that the corresponding volume of the momentum $d P_{o}$ transforms as:

$$
d P_{s}=\gamma d P_{o}
$$


as we have intuitively proposed in Equation (53). Therefore, we can anticipate that a formalism using the technique of Section 2.1 will permit us to obtain formally Equation (60). However, in this subsection will not use Equation (60) and all the result in the following will be obtained just by applying the theory of canonical transformations. Finally, for this reason,

$$
d V d P=d V_{o} d P_{o}
$$

for a one particle phase space. This will be applied in Section 3.3, in order to explain Equation (60). An observable $\langle g\rangle_{s}$ in the moving frame $K$,

$$
\langle g\rangle_{s}=\int g f(q, p, s) d q_{s} d p_{s}
$$

It is important to notice that following Curie et al. [20]

$$
\langle g\rangle_{o}=\int g f(q, p, 0) d q_{o} d p_{o}=\int(\exp [\mathcal{K} s] g) \exp (\mathcal{K} s) f(q, p, 0) d q_{s} d p_{s}
$$

which will represent an important result in order to calculate some observables. As we have noticed before, by using canonical transformation theory for a non-interacting system, Balescu [6] has obtained the distribution function in a moving frame. By starting with the distribution function in the rest frame,

$$
f(q, p, 0)=\frac{1}{Z} \exp \left(-\frac{H}{k T_{o}}\right)
$$

and by applying the boost transformation, we obtain

$$
\begin{aligned}
f(q, p, s) & =\exp (\mathcal{K} s) f(q, p, 0) \\
& =\frac{1}{Z} \exp (\mathcal{K} s) \exp \left(-\frac{H}{k T_{o}}\right)
\end{aligned}
$$

By using the Poisson brackets [6,13],

$$
[\mathcal{K}, P]=H, \quad[\mathcal{K}, H]=P \quad \text { and } \quad[H, P]=0
$$

we arrive at

$$
f(q, p, s)=\frac{1}{Z} \exp \left(\frac{\gamma}{k T_{o}}(H-u P)\right)
$$

We can now calculate one of the more important function in Statistical Mechanics which is the partition function $Z_{o}$, (we have been using just $Z$ without the subscript " $o$ " because we will see that is an invariant as it is expected)

$$
Z_{o}=\frac{1}{n ! h^{3 n}} \int f(q, p, 0) d q_{o} d p_{o}=\frac{1}{n ! h^{3 n}} \int \exp \left(\frac{H}{k T_{o}}\right) d q_{o} d p_{o}
$$

where the factor $1 /\left(n ! h^{3 n}\right)$ has been introduced. If we want to calculate the partition function in the moving frame, $Z_{s}$, we have

$$
Z_{s}=\frac{1}{n ! h^{3 n}} \int f(q, p, s) d q_{s} d p_{s}=\frac{1}{n ! h^{3 n}} \int \exp \left(\frac{\gamma}{k T_{o}}(H-u P)\right) d q_{s} d p_{s}
$$


In order to calculate $Z_{s}$, Balescu [6] committed the mistake of making a change of variable, By using Equations (42) and (43) he arrived at

$$
Z_{s}=\frac{1}{n ! h^{3 n}} \int f(q, p, s) d q_{s} d p_{s}=\frac{1}{n ! h^{3 n}} \int \exp \left(\frac{H}{k T_{o}}\right) \gamma^{-n} d q_{o} \gamma^{n} \prod_{i=1}^{n}\left(1-u \frac{p_{i}}{E_{i}}\right) d p_{o}
$$

where $\gamma^{-n}$ has appeared due to the transformation of the volumes and $p_{i}$ and $E_{i}$ represent the momentum and the energy of each particle. On the other hand, due to the odd character of the term $u(p / E)$, the integral vanishes. Therefore,

$$
Z_{s}=\frac{1}{n ! h^{3 n}} \int \exp \left(\frac{H}{k T_{o}}\right) d q_{o} d p_{o}=Z_{o}
$$

which is equal to the $Z_{o}$. However, as we have seen, it is a mere coincidence of an incorrect procedure. The correct procedure is the following,

$$
\begin{aligned}
Z_{s} & =\frac{1}{n ! h^{3 n}} \int f(q, p, s) d q_{s} d p_{s} \\
& =\frac{1}{n ! h^{3 n}} \int \exp \left(\frac{\gamma}{k T_{o}}(H-u P)\right) d q_{s} d p_{s} \\
& =\int 1 \exp (\mathcal{K} s) \exp \left(\frac{H}{k T_{o}}\right) d q_{s} d p_{s}
\end{aligned}
$$

By noticing that

$$
1=\exp (\mathcal{K} s) 1
$$

we arrive at

$$
Z_{s}=\frac{1}{n ! h^{3 n}} \int(\exp (\mathcal{K} s) 1)\left(\exp (\mathcal{K} s) \exp \left(\frac{H}{k T_{o}}\right)\right) d q_{s} d p_{s}
$$

By using Equation (65), we have

$$
Z_{s}=\frac{1}{n ! h^{3 n}} \int 1 \exp \left(\frac{H}{k T_{o}}\right) d q_{o} d p_{o}=\int \exp \left(\frac{H}{k T_{o}}\right) d q_{o} d p_{o}
$$

Therefore the partition function is an invariant as it has been expected. Therefore, we do not need to put a subscript " $O$ " on the partition function; that is:

$$
Z_{o}=Z_{s}=Z
$$

Let us postpone the proof of the invariance of the distribution function at the end of this subsection.

Let us see a specific example. One of the most important quantities is the energy. Nevertheless, normally molecular interactions with the surface is neglected in statistical mechanics and since the energy transforms as $[12,18]$

$$
E=\gamma\left(E_{\text {rest }}+\frac{u^{2}}{c^{2}} \mathbb{P} V_{\text {rest }}\right)
$$

and

$$
P=\left(\mathbb{P} V_{\text {rest }}+E_{\text {rest }}\right) \gamma \frac{u}{c}
$$


the expression for the energy will be

$$
E=\int H \exp \left(-\frac{\gamma}{k T_{o}}[H-u P]\right) d q_{s} d p_{s}
$$

Nevertheless, this scheme will not allow us to obtain a well-defined quantity since it is obvious that Equation (81) will not involve the pressure $\mathbb{P}$. However, the well-defined quantity is the relativistic redefined energy $\xi=E-u P$ and it is expressed as

$$
\begin{aligned}
\xi & =\frac{1}{Z} \int(H-u P) \exp \left(-\frac{\gamma}{k T_{o}}(H-u P)\right) d q_{s} d p_{s} \\
& =\frac{1}{Z} \int \frac{\gamma}{\gamma}(H-u P) \exp \left(-\frac{\gamma}{k T_{o}}(H-u P)\right) d q_{s} d p_{s} \\
& =\frac{1}{\gamma Z} \int \gamma(H-u P) \exp \left(-\frac{\gamma}{k T_{o}}(H-u P)\right) d q_{s} d p_{s}
\end{aligned}
$$

By using Equation (65), and knowing that

$$
\exp ((K) s) H=\gamma(H-u P)
$$

we obtain,

$$
\begin{aligned}
\xi & =\frac{1}{\gamma Z} \int \gamma(H-u P) \exp \left(-\frac{\gamma}{k T_{o}}(H-u P)\right) d q_{s} d p_{s} \\
\xi & =\frac{1}{\gamma Z} \int(\exp ((K) s) H)\left(\exp (\mathcal{K} s) \exp \left(-\frac{H}{k T_{o}}\right)\right) d q_{s} d p_{s} \\
& =\frac{1}{\gamma Z} \int H \exp \left(-\frac{H}{k T_{o}}\right) d q_{o} d p_{o} \\
& =\gamma^{-1} E_{o}
\end{aligned}
$$

Having a well-defined invariant partition function and the way of obtaining the observables as the redefined energy, we can conclude that the statistical mechanics developed by Balescu [6] is correct provided it is related with the RRT. Therefore, the temperature, in this context must transform as in the RRT. Let us prove it by noticing that the entropy is an invariant. We know that the entropy, $S$,

$$
S=\langle k \ln f\rangle
$$

By using the invariance of the distribution function (we just promise to prove it at the end of the subsection) and by using the invariance of the phase space, it is clear that the entropy, $S$, is also an invariant as it is expected. On the other hand, the temperature $T_{o}$ is obtained by

$$
\frac{\partial S}{\partial E_{o}}=\frac{1}{T_{o}}
$$

If we want to conserve the relation in the moving frame, we need to use the redefined energy, and the result must be

$$
\frac{\partial S}{\partial \xi}=\frac{1}{T}
$$


It has to be noticed that Equation (87) could be considered as an assumption since other choices appear possible. Nevertheless, we have to remember that we have chosen the PE case (see last comment before Section 3.1), and in this case the transformation laws of the thermodynamical quantities indicate that Equation (87) must be satisfied [18]. Therefore, the assumption was to choose the PE case. It should be noted that other choice of the volume, that is other choice of $w^{\mu}$, will obligate us to propose another equation. Looking back to the Equation (69), $\xi=\gamma^{-1} E_{o}$,

$$
\frac{\partial S}{\partial \xi}=\gamma \frac{\partial S}{\partial E_{o}}=\frac{\gamma}{T_{o}}
$$

We can conclude that in the moving frame, the temperature transforms as

$$
T=\gamma^{-1} T_{o}
$$

Therefore, the distribution function, maybe written

$$
f(q, p, s)=\frac{1}{Z} \exp \left(-\frac{1}{k T}(H-u P)\right)
$$

that is,

$$
f(q, p, s)=\frac{1}{Z} \exp \left(-\frac{\Pi}{k T}\right)
$$

where $\Pi=H-u P$. As for the thermodynamics quantities $\xi, T, E_{o}$ and $T_{o}$ which satisfy

$$
\frac{\xi}{T}=\frac{\gamma^{-1} E_{o}}{\gamma^{-1} T_{o}}=\frac{E_{o}}{T_{o}}
$$

we have

$$
\frac{H}{T_{o}} \rightarrow \frac{\Pi}{T}
$$

Therefore, since we know

$$
\int f(q, p, s) d q_{s} d p_{s}=\int f(q, p, 0) d q_{o} d p_{o}
$$

At first glance, we may think that the distribution function is an invariant but this would imply that Equation (60) has been used. That is:

$$
f(q, p, s)=f(q, p, 0)
$$

if Equation (60) is taken into account. In this order ideas, the last identity has to be interpreted as follows:

$$
\begin{aligned}
\frac{1}{Z} \exp \left(-\frac{1}{k T}[H-u P]\right) & =f(q, p, s)=f\left(q_{s}, p_{s}\right) \\
& =f(q, p, 0)=f\left(q_{o}, p_{o}\right) \\
& =\frac{1}{Z} \exp \left(-\frac{H_{o}}{k T_{o}}\right)
\end{aligned}
$$


where $H$ represents the Hamiltonian as function of $q_{s}$ and $p_{s}$, the coordinates and momentums in the $K$ frame and $H_{o}$ the Hamiltonian as a function of $q_{o}$ and $p_{o}$ in the $K_{o}$ frame. As a summary, we present the rules for the boost canonical transformation,

$$
\begin{gathered}
H\left(q_{o}, p_{o}\right) \rightarrow H\left(q_{s}, p_{s}\right)-u P=\Pi\left(q_{s}, p_{s}\right) \\
T_{o} \rightarrow \gamma^{-1} T_{o}=T \\
d q_{o} d p_{o} \rightarrow d q_{s} d p_{s} \\
\exp -\frac{H\left(q_{o}, p_{o}\right)}{k T_{o}} \rightarrow \exp \frac{1}{k T}\left(H\left(q_{s}, p_{s}\right)-u P\right)=\exp \frac{1}{k T} \Pi\left(q_{s}, p_{s}\right)
\end{gathered}
$$

The distribution function expressed by using Equation (91), suggests that we can describe it in a covariant form.

It has to be noticed that Balescu [6] includes in his expression the Helmholtz free energy but we prefer to express the distribution function with the partition function $Z$.

\subsection{Equivalence between the Balescu Statistical Mechanics and the Covariant Redefined Statistical Mechanics}

As we noticed at the end of the last subsection, we must be interested in obtaining a covariant relativistic expression for the distribution function. We must begin by defining the phase space. We saw in Section 2.1, Equation (1), and in Section 3.2, Equations (60) and (62) which can be written as

$$
\begin{aligned}
d V & =\frac{1}{w^{\mu} u_{\mu}} d V_{o}, \quad d P=w^{\mu} u_{\mu} d P_{o}, \\
& \Rightarrow \quad d V d P=\frac{1}{w^{\mu} u_{\mu}} d V_{o} w^{\mu} u_{\mu} d P_{o}=d V_{o} d P_{o}
\end{aligned}
$$

Therefore, the phase space can be now expressed without any subscript, that is

$$
d q d p=d q_{s} d p_{s}=d q_{o} d p_{o}
$$

Nevertheless, we need to indicate the reference frame where the volume is considered. Therefore, we shall denote the phase space by $d \Gamma_{s}$ where the subscript " s" specifies the choice of the volume. By correspondence with Equation (20), Let us define the $4-$ vector $\Pi^{\mu}$ as

$$
\Pi^{\mu}=\frac{w^{\mu}}{w^{\mu} u_{\mu}} H
$$

It is easy to see that, in our choice of the volume, the PE case,

$$
\Pi^{0}=H-u P
$$

We also have,

$$
\beta_{\mu} \Pi^{\mu}=\frac{u_{\mu}}{k T_{o}} \frac{w^{\mu}}{w^{\lambda} u_{\lambda}} H\left(q_{o}, p_{o}\right)=\frac{H\left(q_{o}, p_{o}\right)}{k T_{o}}=\frac{1}{k T}\left(H\left(q_{s}, p_{s}\right)-u P\right)
$$

where we have used the $4-$ vector temperature, $u_{\mu} /\left(k T_{o}\right)$. Therefore, the distribution function can be expressed as:

$$
f=\frac{1}{Z} \exp -\beta_{\mu} \Pi^{\mu}
$$


Therefore, the formalism of canonical transformation along with the formalism of Section 2.1, permit us to understand the meaning of such a theory. The distribution function can be expressed in an invariant form, Equation (103), and the transformation of $d p d q$ does not correspond to a change of variable nor a Lorentz transformation which will have no meaning due to the simultaneity effect, but to a change of canonical factors as it is expressed in Equations (98) and (99). It has to be pointed out that for the PE case

$$
d V_{s}=\gamma^{-1} d V_{o} \quad \text { and } \quad d P_{s}=\gamma d P_{o}
$$

while for the RG case

$$
d V_{s}=\gamma d V_{o} \quad \text { and } \quad d P_{s}=\gamma^{-1} d P_{o}
$$

Equation (103) shows the invariance character of the distribution function. Any well-defined observable can be now expressed as

$$
\langle g\rangle_{s}=\frac{1}{Z} \int g \exp -\beta_{\mu} \Pi^{\mu} d \Gamma_{s}
$$

This means that we have to take the representation of $\beta_{\mu} \Pi^{\mu}$ as

$$
\beta_{\mu} \Pi^{\mu}=\frac{1}{k T}\left(H\left(q_{s}, p_{s}\right)-u P\right)
$$

because we are dealing with the phase space $d \Gamma_{s}$. However, we can change to

$$
\begin{aligned}
\langle g\rangle_{s} & =\frac{1}{Z} \int g \exp \mathcal{K} s \exp -\frac{H}{k T_{o}} d \Gamma_{o} \\
\langle g\rangle_{s} & =\frac{1}{Z} \int(\exp (-\mathcal{K} s) g) \exp -\frac{H}{k T_{o}} d \Gamma_{o}
\end{aligned}
$$

It has to be noticed that the boost canonical transformation of $g$, (see Curie et al. [20] Section III)

$$
\exp (\mathcal{K} s) g(q, p)=g(\exp (\mathcal{K} s) q, \exp (\mathcal{K} s) p)
$$

In other words, a canonical transformation on the functions on phase space can be represented by the canonical transformation of the phase space. It has to be noticed that when the observable is an invariant,

$$
\exp (-\mathcal{K} s) g(q, p)=g(\exp -(\mathcal{K} s) q, \exp -(\mathcal{K} s) p)=g\left(q_{o}, p_{o}\right)
$$

as it is the case for the partition function, $Z$, since $\exp ((\mathcal{K}) s) 1=1$.

On the other hand, if the function transformed as

$$
\exp (-\mathcal{K} s) g(q, p)=\gamma^{-1} g\left(q_{o}, p_{o}\right)
$$

Considering that in the integral the subscript " $O$ " is not necessary, we have

$$
\begin{aligned}
\langle g\rangle_{s} & =\frac{1}{Z} \int g \exp -\beta_{\mu} \Pi^{\mu} d \Gamma_{s}=\int \gamma^{-1} g(q, p) \frac{1}{Z} \exp -\frac{H}{k T_{o}} d \Gamma_{o} \\
& =\gamma^{-1} \frac{1}{Z} \int g(q, p) \exp -\frac{H}{k T_{o}} d \Gamma_{o} \\
& =\gamma^{-1}\langle g\rangle_{o}
\end{aligned}
$$


This is the case of $\Pi=E-u P$. Indeed,

$$
\exp -(\mathcal{K} s)(H-u P)(q, p)=\gamma^{-1} H\left(q_{o}, p_{o}\right)
$$

Therefore in this case, by using Equation (106), we have

$$
\begin{aligned}
\xi & =\langle H-u P\rangle_{s}=\frac{1}{Z} \int(\exp (-\mathcal{K} s)(H-u P)) \exp -\frac{H}{k T_{o}} d \Gamma_{o} \\
& =\gamma^{-1} \frac{1}{Z} \int H \exp -\frac{H}{k T_{o}} d \Gamma_{o}=\gamma^{-1} E_{o}
\end{aligned}
$$

as it was expected by the RRT. In the case of the energy, we do not have this behavior of the energy function and consequently it will not represent a well-defined quantity in Relativistic Thermodynamics.

Finally, we can affirm that the covariant expression of the distribution function described by Equation (103) is equivalent to the Balescu relativistic proposal [6] and both theories generate the RRT. Of course, the 4-inverse temperature, (24), and the covariant expressions, Equations (102) and (103), has been used before [15-17] but without relating them with the RRT.

\subsection{The Jüttner Distribution Function in a Moving Frame}

In the beginning of the last century Jüttner [3] derived an equilibrium distribution function $f(p)$ for a relativistic ideal gas. Since the distribution function has been used in many applications, it represents a cornerstone of Relativistic Statistical Physics. Although many authors have doubt about the validity of it, we can cite the work done by Horwitz [19] among others, Debbasch [23] have proved the correctness of the Jüttner distribution function.

Even if we have proved that from the Covariant Redefined Statistical Mechanics, we can deduce the RRT, it is important to deduce the transformed Js. That is, we choice the PE volume; in other words, the volume is considered in the $K$ system and the simultaneity is in $K$. Therefore,

$$
\begin{aligned}
T & =\gamma^{-1} T_{o} \\
\beta_{\mu} H^{\mu} & =\frac{1}{k T}(H-u P)=\frac{1}{k T}\left(\sum_{i=1}^{n} \sqrt{p_{i}^{2} c^{2}+m^{2} c^{4}}-u P\right)
\end{aligned}
$$

Firstly, let us calculate the distribution function in one dimension (even in one dimension, the one dimensional volume will be called $V$ or $V_{o}$ ), that is

$$
f(q, p)_{s}=\frac{1}{Z} \exp -\frac{1}{k T}\left(\left(\sum_{i=1}^{n} \sqrt{p_{i}^{2} c^{2}+m^{2} c^{4}}\right)-u P\right)
$$

The relativistic Js is expressed by (see Equation (7.77) of Greiner)

$$
f(\vec{p})=\frac{1}{Z} \int \sum_{i=1}^{n}\left(\delta\left(\vec{p}_{i}-\vec{p}\right) \exp -\frac{1}{k T}\left(\left(\sum_{i=1}^{n} \sqrt{p_{i}^{2} c^{2}+m^{2} c^{4}}\right)-P u\right) d V^{n} d p^{n}\right.
$$

where

$$
P=\sum_{i=1}^{n} p_{i}
$$


Therefore,

$$
f(\vec{p})=\frac{1}{Z} \int \sum_{i=1}^{n}\left(\delta\left(\vec{p}_{i}-\vec{p}\right) \exp -\frac{1}{k T}\left(\left(\sum_{i=1}^{n} \sqrt{p_{i}^{2} c^{2}+m^{2} c^{4}}-p_{i} u\right)\right) d V^{n} d p^{n}\right.
$$

by separating each integral, we have

$$
\begin{aligned}
f(\vec{p}) & =\frac{n}{Z} \prod_{i=2}^{n}\left(\int \exp -\frac{1}{k T}\left(\sqrt{p_{i}^{2} c^{2}+m^{2} c^{4}}-p_{i} u\right) d V d p_{i}\right) \\
& \times V \exp -\frac{1}{k T}\left(\left(\sum_{i=1}^{n} \sqrt{p^{2} c^{2}+m^{2} c^{4}}-p u\right)\right)
\end{aligned}
$$

Let us apply the same form to the partition function, $Z$, that is:

$$
\begin{aligned}
Z & =\frac{1}{n ! h^{n}} \int \exp -\frac{1}{k T}\left(\left(\sum_{i=1}^{n} \sqrt{p_{i}^{2} c^{2}+m^{2} c^{4}}\right)-P u\right) d V^{n} d p^{n} \\
& =\frac{1}{n ! h^{n}}\left(\int \exp -\frac{1}{k T}\left(\left(\sqrt{p_{1}^{2} c^{2}+m^{2} c^{4}}\right)-p_{1} u\right) d V d p_{1}\right) \\
& \times \prod_{i=2}^{n}\left(\int \exp -\frac{1}{k T}\left(\left(\sqrt{p_{i}^{2} c^{2}+m^{2} c^{4}}\right)-p_{i} u\right) d V d p_{i}\right)
\end{aligned}
$$

The term $h^{n}$ is due to the choice of working in one dimension. The first part of Equation (119) is equal to:

$$
\begin{aligned}
& \frac{1}{h} \int \exp -\frac{1}{k T}\left(\left(\sqrt{p_{1}^{2} c^{2}+m^{2} c^{4}}\right)-p_{1} u\right) d V d p_{1} \\
& =\frac{1}{h} \int \exp \left(-\frac{1}{k T_{o}}\left(\sqrt{p_{o 1}^{2} c^{2}+m^{2} c^{4}}\right)\right) d V_{o} d p_{o 1}=V_{o} \Upsilon_{1}\left(T_{o}\right)
\end{aligned}
$$

The partition function can be written as

$$
Z=\frac{1}{n !}\left(V_{o} \Upsilon_{1}\left(T_{o}\right)\right)^{n}
$$

Therefore, by using Equations (117), (119), (120) and (121) we arrive at

$$
\begin{aligned}
f(\vec{p}) & =\frac{V}{V_{o} \Upsilon_{1}\left(T_{o}\right)} \exp -\frac{1}{k T}\left(\left(\sqrt{p^{2} c^{2}+m^{2} c^{4}}\right)-p u\right) \\
& =\frac{1}{\gamma(u) \Upsilon\left(T_{o}\right)} \exp -\frac{\gamma}{k T_{o}}\left(\left(\sqrt{p^{2} c^{2}+m^{2} c^{4}}\right)-p u\right)
\end{aligned}
$$

If we want to express the distribution in terms of the rest temperature $T_{o}$, the velocity $v$ of the particle in $K$ and the velocity $u$ of $K$, we have

$$
\begin{aligned}
f(v) & =\frac{1}{\gamma(u) \Upsilon\left(T_{o}\right)} \exp -\frac{\gamma(u)}{k T_{o}}(m \gamma(v)-m \gamma(v) v u) \frac{d p}{d v} \\
& =\frac{1}{\gamma(u) \Upsilon\left(T_{o}\right)} \exp -\frac{m \gamma(u) \gamma(v)}{k T_{o}}(1-v u) \frac{d p}{d v}
\end{aligned}
$$

We know that

$$
\frac{d p}{d v}=m \gamma^{3}
$$


Finally, the distribution function is:

$$
f(v)=\frac{m \gamma^{3}(v)}{\gamma(u) \Upsilon_{1}\left(T_{o}\right)} \exp -\frac{m \gamma(u) \gamma(v)}{k T_{o}}(1-v u)
$$

which coincides with the expression proposed by Cubero et al. [21] and Dunkel et al. [24,25] for the case in one dimension and the difference in the sign is due to considering the moving frame as coming and not going.

On the other hand, if we want to express the result in three dimension, we just have to include the geometry of the transformation by using $\vec{v}=v_{x} \widehat{i}+v_{y} \widehat{j}+v_{z} \widehat{k}$ and the fact that

$$
f(\vec{p}) d p^{3}=f(\vec{v}) d v^{3}
$$

that is,

$$
f(\vec{v})=f(\vec{p}) \frac{\partial p_{x} p_{y} p_{z}}{\partial v_{x} v_{y} v_{z}}
$$

Being

$$
p_{x}=m \gamma(v) v_{x} \quad p_{y}=m \gamma(v) v_{y} \quad \text { and } \quad p_{z}=m \gamma(v) v_{z}
$$

we arrive at

$$
J=\frac{\partial p_{x} p_{y} p_{z}}{\partial v_{x} v_{y} v_{z}}=m^{3} \gamma^{5}
$$

Finally, it is easy to see that in three dimension the Js can be expressed as

$$
f(\vec{p})=\frac{V}{V_{o} \Upsilon_{3}\left(T_{o}\right)} \exp -\frac{1}{k T}\left(\left(\sqrt{p^{2} c^{2}+m^{2} c^{4}}\right)-\vec{p} \cdot \vec{u}\right)
$$

where

$$
\Upsilon_{3}\left(T_{o}\right)=\frac{4 \pi}{h^{3}}(m c)^{3} \frac{K_{2}\left(\frac{m c^{2}}{k T_{o}}\right)}{m c^{2}} k T_{o}
$$

being $K_{2}$ the Bessel function of second rank. If we want to express Js as a function of the velocity, we have

$$
f(\vec{v})=\frac{m^{3} \gamma^{5}(\vec{v})}{\gamma(\vec{u}) \Upsilon_{3}\left(T_{o}\right)} \exp -\frac{m \gamma(\vec{u}) \gamma(\vec{v})}{k T_{o}}(1-\vec{v} \cdot \vec{u})
$$

The minus sign appears due to considering the system as coming and not going. Nevertheless, if we are measuring in the reference $K$, our thermometer will detect the temperature in such a frame, that is: $T=\gamma^{-1} T_{o}$. Therefore, Equation (132) can be written in term of the temperature in the $K$ frame, as

$$
f(\vec{v})=\frac{m^{3} \gamma^{5}(\vec{v})}{\gamma(\vec{u}) \Upsilon_{3}(\gamma(\vec{u}) T)} \exp -\frac{m \gamma(\vec{u}) \gamma(\vec{v})}{k T}(1-\vec{v} \cdot \vec{u})
$$

Equations (132) and (133) represent the Js and coincide with the rest Jüttner distribution function when $u=0$.

\section{The Interacting Case}

As we notice in the introduction, due to the difficulty of formulating a relativistically consistent Hamiltonian approach for the interacting particles case [20], it is prohibited to use the boost transformation of the distribution function as $f(q, p, s)=\exp (\mathcal{K} s) f(q, p, 0)$, Equation (58). Therefore, 
all the Relativistic Statistical Mechanics described in Section 3.3 are not valid for the interacting case. However, the RRT is independent of the no-interaction theorem. The results obtained in Section 2 represent a well-defined theory which is covariant and consistent. That is, Equations (14), (23), (24), (25), (32) and all the results of the Section 2 are valid. Nevertheless, everything is based on the assumption that we can define a rest frame for each equilibrium system. However, the essential problem of defining a center of motion is still present for a system of interacting particles. Indeed, in a seminal paper in 1948, Pryce [22] tried to identify the natural definition of the center-of-mass for a relativistic system of particles. He suggested that a many-particle relativistic dynamics can be constructed provided that we give up the assumption of invariant world-lines. The real problem facing any attempt to construct a relativistic many-particle theory is the lack of a clear notion of simultaneity. Later, Bakamjian and Thomas [44] constructed the canonical center-of-mass and the relative particle position and momentum vectors with respect to the center-of-mass, explicitly producing the canonical transformation required but the Minkowski world-line postulate was rejected. A search of the literature shows that the work of Bakamjian and Thomas [44,45] was ignored until 1963, when Currie, Jordan, and Sudarshan [20] proved the well-known no-interaction theorem explicitly showing that the conjecture of Thomas was true. Nevertheless, the problem of defining a rest frame for a system of particles is still an open problem. Recently, some interesting attempts have been proposed by many authors. In particular, the work done by Gill and Zachary [30] gives a definition of the center of mass by using a new concept, the canonical proper time. Although the Minkowski world-line postulate is not considered, the theory possesses the interesting property of defining the concept of simultaneity for all the particles of the system. This idea coincides with Lehmann proposal [26] of defining a universal parameter for obtaining a covariant statistical theory.

Moreover, even if the RRT seems to be not affected by the no-interaction theorem, as we noticed above, it is based on the assumption that the rest frame of a system of particles can be defined. Fortunately, the RRT itself indicates us which is the rest frame. The idea consists of noticing that the RRT predicts that the temperature is a maximum when it is measured in the rest frame of the system. In fact, $T=\gamma^{-1} T_{o}$ (see Table 1 and Equation (86)) represents the transformation law of the temperature from the rest frame $K_{o}$ to the frame $K$. In the system $K$, the laws of thermodynamics are valid as we have showed in Section 2 and in other papers [18]. Therefore, we just need to use a thermometer in different frames and find in which reference frame the temperature is the maximum. We will obtain with this method the rest frame of our equilibrium thermodynamical system. Fortunately, a blackbody in each reference frame can be used as a thermometer [18,46]. Indeed, a perfect Planckian distribution function of the frequencies will indicates us that the blackbody is at rest with respect our system. A deviation of the Planckian form will indicate the speed of the rest frame and the temperature of the blackbody (for more information about the use of a blackbody as a relativistic thermometer see [18,46]). Moreover, the study of relativistic equilibrium systems is related to astrophysics, as for example plasmas at high temperature moving with respect our detectors. Normally, the systems are themselves blackbodies or are very close to them and consequently we just need to know its frequency radiation in order to know their motion, their temperature and finally their rest frame. 
Therefore, thermodynamical quantities in a moving frame can be obtained from the rest quantities. For example the redefined energy

$$
\xi=\frac{w^{0}}{w^{\lambda} u_{\lambda}} E_{o}=\gamma^{-1} E_{o}
$$

where $E_{o}$ is calculated in the rest frame by using the regular Relativistic Statistical Mechanics.

$$
E_{o}=\int H \exp -\frac{H}{k T_{o}} d \Gamma_{o}
$$

Therefore, we do not need to use canonical transformations which are not allowed by the no-interaction theorem, in order to obtain the relativistic thermodynamical quantities in a moving frame. In fact, Relativistic Statistical Mechanics cannot be obtained for the interacting case due to the no-interacting case, but due to that the RRT a rest frame can be deduced and in this frame all the thermodynamical quantities are gotten and then they can be transformed to the moving frame. The problem is reduced to describe the rest frame which is experimentally possible.

\section{Concluding Remarks}

We have developed a Covariant Relativistic Statistical Mechanics which is compatible with the RRT for the non-interacting case. Moreover, we have showed that although Balescu [6] Statistical Mechanics is not expressed in a covariant form, it gives equivalent results to our covariant theory and also generates the RRT. As a consequence, we can affirm that previous results $[12,13,18]$ obtained in other works without using the covariant form are valid. The Js has been obtained for the 1-dimension case and the regular 3-dimension case. All the properties of the RRT can be deduced from the new statistical theory and the main result is that the new theory is covariant. Nevertheless, the theory is just valid for non-interacting systems. Indeed, the no-interaction theorem does not permit to use the boost canonical transformations in order to obtain the distribution function in a moving frame. However, for the interacting case, a proposal for finding the rest frame of the system has been exposed. Relativistic Statistical Mechanics of a system must be studied in its own rest frame and all the thermodynamical quantities can be obtained in another reference frame by just applying the transformation laws deduced from the RRT.

Finally, it has to be noticed that even if the no-interacting theorem does not permit to deal with a system of particles, the RRT avoids this problem by taking into account the regular concept of temperature.

\section{Acknowledgements}

This work has been partially supported by COFAA and EDI IPN, and CONACYT. 


\section{References}

1. Planck, M. Zur dynamik bewegter systeme. Ann. Phys. 1908, 331, 1-34.

2. Einstein, A. Über das Relativitätsprinzip und die aus demselben gezogenen Folgerungen. Jahrb. Radioakt. Elektron. 1907, 4, 411-462.

3. Jüttner, F. Das maxwellsche gesetz der geschwindigkeitsverteilung in der relativtheorie. Ann. Phys. 1911, 339, 856-882.

4. Landsberg, P.T.; Matsas, G. Laying the ghost of the relativistic temperature transformation. Phys. Lett. A 1996, 223, 401-403.

5. Ott, H. Lorentz-transformtion der warme und der temperatur. Z. Phys. 1963, 175, 70-104.

6. Balescu, R. Relativistic statistical thermodynamics. Physica 1968, 40, 309-338.

7. Sieniutycz, S. Thermodynamic and relativistic aspects of thermal inertia in fluids. Phys. Rev. E 1998, 58, 7027-7039.

8. Landsberg, P.T. Does a moving body appear cool? Nature 1966, 212, 571-572.

9. Landsberg, P.T. Does a moving body appear cool? Nature 1967, 214, 903-904.

10. Landsberg, P.T. Special relativistic thermodynamics. Proc. Phys. Soc. 1966, 89, 571-572.

11. Landsberg, P.T.; Matsas, G. The impossibility of a universal relativistic temperature transformation. Physica A 2004, 340, 92-93.

12. Ares de Parga, G.; López-Carrera, B.; Angulo-Brown, F. A proposal for relativistic transformations in thermodynamics. J. Phys. A Math. Gen. 2005, 38, 2821-2834.

13. López-Carrera, B.; Ares de Parga, G. Relativistic transformation of the canonical distribution function in relativistic statistical mechanics. Physica A 2008, 387, 1099-1109.

14. Nakamura, T.K. Covariant thermodynamics of an object with finite volume. Phys. Lett. A 2006, $352,175-177$.

15. van Kampen, N.G. Relativistic thermodynamics of moving systems. Phys. Rev. 1968, 173, 295-301.

16. Israel, W. Thermodynamics of relativistic systems. Physica A 1981, 106, 204-214.

17. Israel, W. Nonstationary irreversible thermodynamics: A causal relativistic theory. Ann. Phys. 2009, 100, 310-331.

18. Ares de Parga, G.; López-Carrera, B. Redefined relativistic thermodynamics based on the Nakamura formalism. Physica A 2009, 388, 4345-4356.

19. Horwitz, L.P.; Schieve, W.C.; Piron, C. Gibbs ensembles in relativistic classical and quantum mechanics. Ann. Phys. 1981, 137, 306-340.

20. Currie, D.G.; Jordan, T.F.; Sudarshan, E.C.G. Relativistic invariance and hamiltonian theories of interacting particles. Rev. Mod. Phys. 1963, 35, 350-375.

21. Cubero, D.; Casado-Pascual, J.; Dunkel, J.; Talkner, P.; Hänggi, P. Thermal equilibrium and statistical thermometers in special relativity. Phys. Rev. Lett. 2007, 99, 170601.

22. Pryce, M.H.L. The mass-centre in the restricted theory of relativity and its connexion with the quantum theory of elementary particles. Proc. R. Soc. Lond. Ser. A Math. Phys. Sci. 1948, $195,62-81$. 
23. Debbasch, F. Equilibrium distribution function of a relativistic dilute perfect gas. Physica A 2008, 387, 2443-2454.

24. Dunkel, J.; Talkner, P.; Hänggi, P. Relative entropy, Haar measures and relativistic canonical velocity distribution. New J. Phys. 2007, 9, Progressive number 144.

25. Dunkel, J.; Hänggi, P. One-dimensional non-relativistic and relativistic Brownian motions: A microscopic collision model. Physica A 2007, 374, 559-572.

26. Lehmann, E. Covariant equilibrium statistical mechanics. J. Math. Phys. 2006, 47, Progressive number 023303.

27. Requardt, M. Thermodynamics meets special relativity-or what is real in physics? arXiv 2008, arXiv:0801.2639v1 [gr-qc].

28. Möller, C. The Theory of Relativity, 2nd ed.; Clarendon Press: Oxford, UK, 1972; Chapter 7.

29. Nakamura, T.K. Relativistic energy-momentum of a body with a finite volume. Space Sci. Rev. 2006, 122, 271-278.

30. Gill, T.L.; Zachary, W.W. Two mathematically equivalent versions of maxwell's equations. Found. Phys. 2009, 41, 99-128.

31. Staruszkiewicz, A. Relativistic transformation laws for thermodynamical variables with applications to classical electron theory. Il Nuovo Cimento A 1966, XLV, 6632-6636.

32. Rohrlich, F. True and apparent transformations, classical electrons, and relativistic thermodynamics. Il Nuovo Cimento B 1966, XLV, 6200-6207.

33. Fermi, E. Correzione di una contraddizione tra la teoria elettrodinamica e quella relativistica delle masse elettromagnetiche. Il Nuovo Cimento 1923, 25, 159-170.

34. Synge, J.L. Relativity, the Special Theory; North-Holland Publishing Co.: Amsterdam, The Netherlands, 1965; pp. 429-430. Appendix D.

35. Rohrlich, F. Classical Charged Particles. Foundations of Their Theory. In Addison-Wesley Series in Advanced Physics; Addison-Wesley: Boston, MA, USA, 1965; p. 282. Appendix A1-6.

36. Gamba, A. Physical quantities in different reference systems according to relativity. Am. J. Phys. 1967, 35, 83-88.

37. Landau, L.; Lifshitz, E. The Classical Theory of Fields; Pergamon Press: Oxford, UK, 1962; Volume 2, Chapter 32.

38. Weinberg, S. Gravitation and Cosmology, Principles and Applications of the General Theory of Relativity; Wiley: Hoboken, NJ, USA, 1972; p. 48.

39. Balescu, R.; Kotera, T. On the covariant formulation of classical relativistic statistical mechanics. Physica 1967, 33, 558-580.

40. Hakim, R. Remarks on relativistic statistical mechanics I. J. Math. Phys. 1967, 8, 1315-1344.

41. Hakim, R.; Mangeney, A. Relativistic kinetic equations including radiation effects, I. Vlasov approximation J. Math. Phys. 1968, 9, 116-130.

42. Landau, L.; Lifshitz, E. The Classical Theory of Fields; Pergamon Press: Oxford, UK, 1962; Volume 2, Chapter 6 and 10.

43. van Kampen, N.G. Lorentz-invariance of the distribution in phase space. Physica 1969, 43, 244-262.

44. Bakamjian, B.; Thomas, L.H. Relativistic particle dynamics. II. Phys. Rev. 1953, 92, 1300-1310. 
45. Thomas, L.H. The relativistic dynamics of a system of particles interacting at a distance. Phys. Rev. 1952, 85, 868-872.

46. López-Carrera, B.; Rosales, M.A.; Ares de Parga, G. The 2.7 K black-body radiation background reference frame. Chin. Phys. B 2010, 19, Progressive number 040203.

(C) 2011 by the authors; licensee MDPI, Basel, Switzerland. This article is an open access article distributed under the terms and conditions of the Creative Commons Attribution license (http://creativecommons.org/licenses/by/3.0/.) 